\title{
Low-Cost Android Based Telemonitoring System for Body Temperature Measurement
}

\author{
Ahmet Remzi Ozcan' ${ }^{1}$, Ahmet Mert $^{1 *}$ \\ 1* Bursa Technical University, Bursa, Turkey
}

Cite this paper as:

Ozcan, R. A., Mert, A. (2021). Low-Cost Android Based Telemonitoring System for Body Temperature Measurement. Journal of Innovative Science and Engineering. 5(2): $91-100$

\section{*Corresponding author: Ahmet Mert} E-mail: ahmet.mert@btu.edu.tr

Received Date: 06/12/2020 Accepted Date: 06/02/2021 (C) Copyright 2021 by Bursa Technical University. Available online at http://jise.btu.edu.tr/

\section{cc) (i) (8)}

The works published in Journal of Innovative Science and Engineering (JISE) are licensed under a Creative Commons Attribution-NonCommercial 4.0 International License.

\section{Abstract}

The increasing number of pandemic issues require to pay attention to health conditions and social distance. The explicit sign of COVID-19 is body fever. It is a simple and affordable detection method when compared to other blood tests. However, it is required to be physically close to a visitor to measure body temperature. For this reason, we have designed and developed a low-cost microprocessor measurement system with an infrared non-contact temperature sensor, and a Bluetooth for sending to long distance. Android application has been developed to read and set the alarm function using a smart telephone or a tablet far from the visitors. With the help of these circuit and application designs, body temperatures can be checked from long distance considering the pandemic situations. The printed circuit of the microcontroller, Bluetooth, sensor, and light-dependent resistor (LDR) triggering are manufactured, and the software of the controller and the application are integrated and tested successfully.

Keywords: Temperature sensor, Telemonitoring, Body temperature, Measurement, Android application. 


\section{Introduction}

The increasing number of pandemic issues and chronic illness of the elderly require to pay attention to the health condition. The monitoring physiological values of chronic patients were one of the popular topics in biomedical engineering. With the help of the advanced medical sensor technologies, telemonitoring of medical parameters of the patient is a useful tool for healthcare specialists. Heart rate monitoring based on photoplethysmography (PPG) or electrocardiography (ECG) [1] is the most studied electronic system the telemonitoring system due to being a vital indicator [2]. Blood pressure, electroencephalography (EEG), and blood glucose are also used for long-term monitoring, especially for telemedicine $[3,4]$.

Body temperature is the most applied measurement for the diagnosis of an illness. For this reason, various types of devices have been designed. NTC type thermistor, and contactless IR sensor-based devices are the most common types. Due to fast response and being contactless, IR temperature circuit is preferred [5]. There is an increasing demand for contactless temperature sensor after SARS-CoV-2 pandemic. The fever is the most general symptom, so researchers focus on contactless temperature telemonitoring due to prevent the spread of COVID-19. A wearable device, and measurement from wrist were applied to patients in hospitals to investigate the effects on COVID-19 pandemic [6,7].

Biotelemetry is interdisciplinary engineering to transfer and monitor various vital signs of ambulatory patients [8]. Saturated O2 (SpO2), ECG, body temperature can be connected to the microcontroller and wireless communication systems. Thus, these phycological signals can be traced from long distance or internet [9]. Telemedicine or telemonitoring systems can be categorized depending on measured signal(s) /sign(s), a total number of the channels (mono or multi), communication protocol, and the user interface [10]. After signal amplification, it was applied to the microcontroller for processing and sent via Bluetooth, ZigBee, and Wi-Fi networks [12-14]. Wireless body area networks (WBANs) also combine multi- sensor signal processing and transmitting them via multiple communication systems depending signals health priority [15-17]. Thus, the health status of the patients can be detected, and doctors can track them without visiting the hospital [15]. In [18], GPS was integrated into the measurement system to track the patient. In addition, there are some commercial devices and applications to monitor some phycological signals (e.g, smartwatches), which are listed in [19].

In this study, we designed an IR contactless temperature monitoring circuit and application in accordance with pandemic conditions. The temperature measurement circuit is designed using an IR sensor, microcontroller, and Bluetooth. An Android application is developed to monitor and warn after receiving Bluetooth signals. Thus, body temperature can be checked from a long distance without disturbing isolation in SARS-CoV-2 pandemic. This developed system can be adapted to continuous temperature device in the range of Bluetooth. The remainder of the paper is organized as follows: Section Material and Methods provides the description of the used components, the designed circuit and the application. In Section Results and Discussion designed system and application software are examined, and the conclusions are drawn in Section Conclusion. 


\section{Material and Methods}

\subsection{Non-Contact Temperature Sensor}

Thermopile IR sensors are based on the detection of IR energy from an object from a distance. The more energy means a higher temperature level. We have used the medical version of the MLX90615 IR sensor [20]. It consists of the sensor, a 16-bit ADC, and a microcontroller with SMBus (similar to I2C) protocol. The block diagram of the sensor is given in Figure 1.

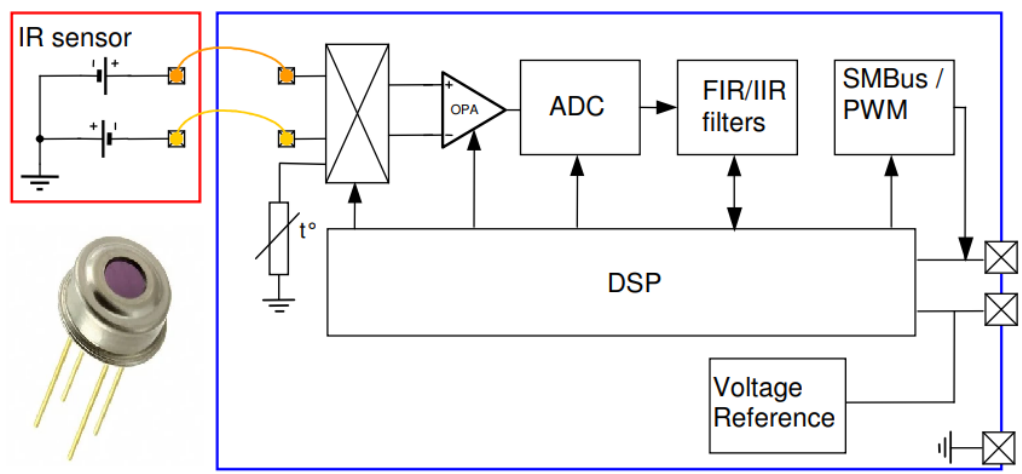

Figure 1. The block diagram of the non-contact temperature sensor, MLX90615 [20].

This sensor has a fast response of measurement with error rate $\pm 0.2^{\circ} \mathrm{C}$ in the medical range shown in Figure 2 .

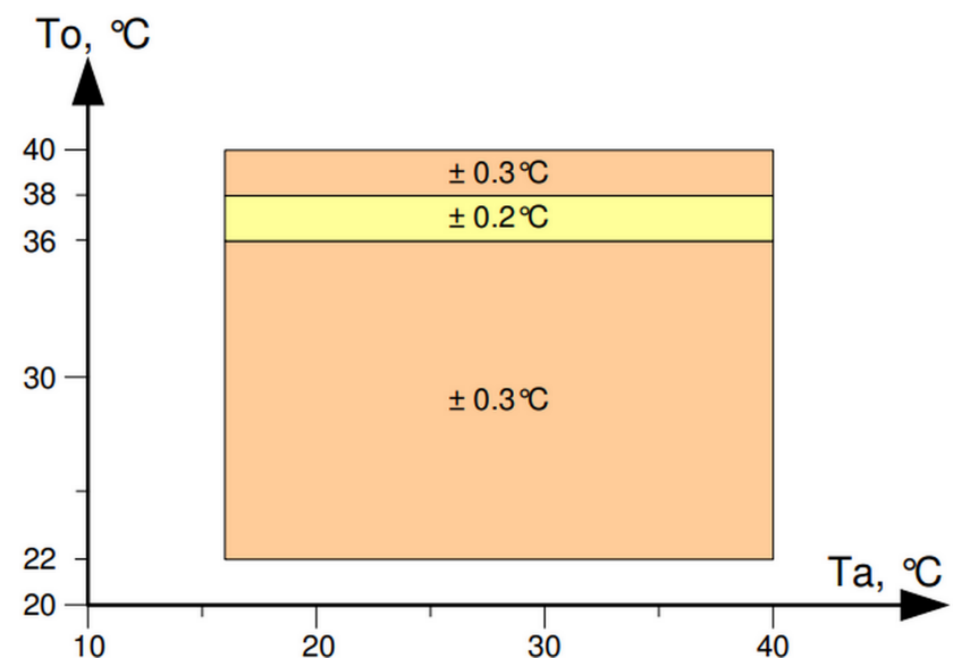

Figure 2. Accuracy of the sensor for medical applications [20].

MLX90615DAA has self-calibrating property depending on ambient temperature (Ta) measured by the internal sensor. The detected temperature of the object (To) can be read from the memory of 0xB7 using two-wire SMBus. 


\subsection{Design of Microcontroller Based Measurement Device}

A circuit consisting of a microcontroller, Bluetooth, LED, light-dependent resistor (LDR), and MLX 90615 IR temperature sensor has been designed in this study. For temperature measurement MLX 90615 is connected to low energy microcontroller, PIC12LF1822 [21] via I2C I/O pins. The measurement starts with the trigger signal from the LDR pin, and then the temperature in the $0 \mathrm{xB} 7$ using $\mathrm{I} 2 \mathrm{C}$ is processed to convert it to Celsius degree. Finally, the value is sent using Bluetooth to an Android application, and the status LED is turned on. The block diagram of the designed circuit is shown in Figure 3.

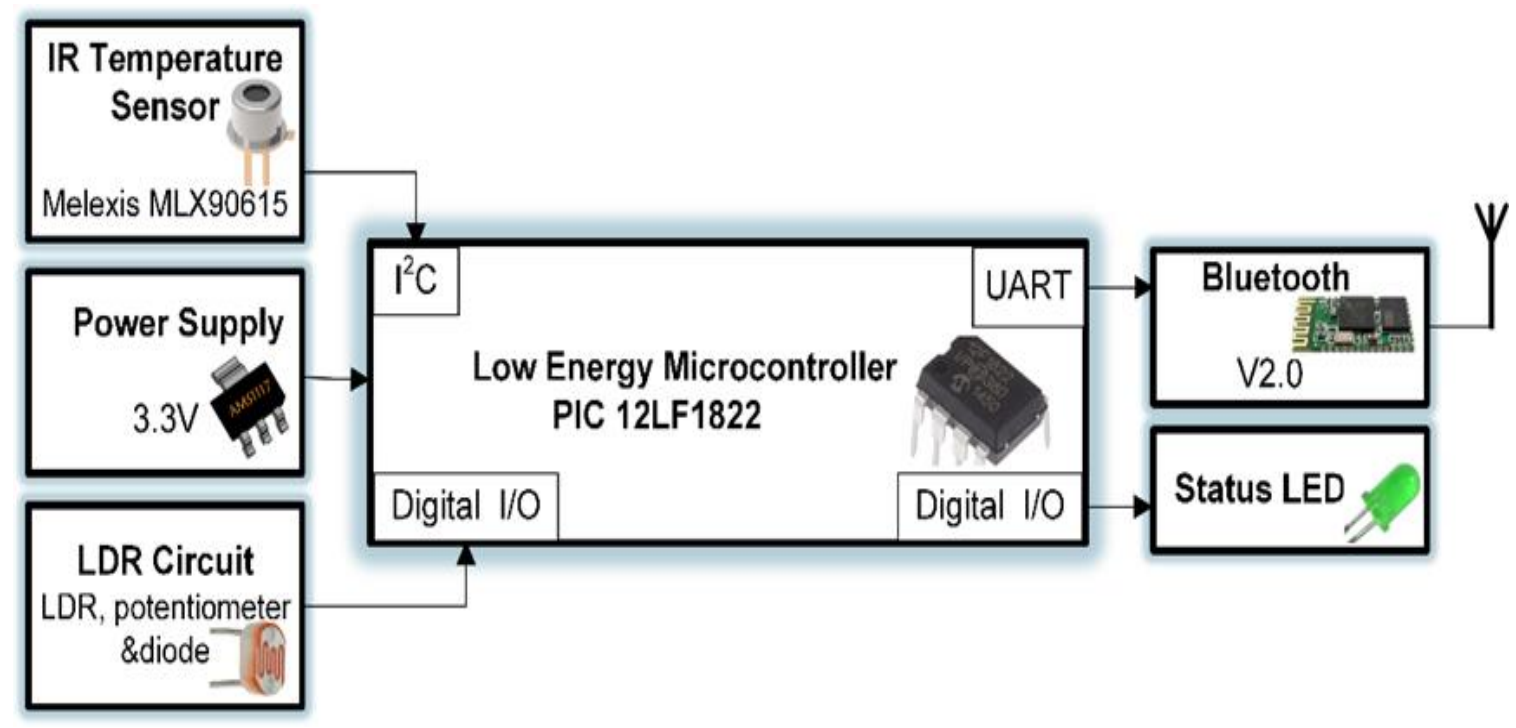

Figure 3. The block diagram of the designed microcontroller-based measurement device.

The LDR circuit is to detect the wrist is placed or not. After detection, the trigger digital level exists, and the measurement starts via $\mathrm{I} 2 \mathrm{C}$. The value in $0 \mathrm{xB} 7$ is then converted by

$$
T\left({ }^{\circ} \mathrm{C}\right)=0 \mathrm{xB} 7 \times 0.02-273.15
$$

The temperature is transferred writing it to UART port of the controller, and then it is received by the paired Bluetooth module of the Android device. An algorithm is also developed for PIC12LF1822 microcontroller, and the pseudocode is given in Algorithm 1. 
Algorithm 1: The Pseudo-Code of The Microcontroller.

Program starts

Initialize Configuration,

Initialize UART module at $9600 \mathrm{bps}$,

Initialize $\mathrm{I}^{2} \mathrm{C}$ module at $100 \mathrm{kHz}$.

\section{Start Main Loop}

Start Loop1

Send ID via UART

IF Sent ID==Received ID Then Break

End Loop1

IF LDR Pin==True Then

WakeUp MLX90615
Start $\mathrm{I}^{2} \mathrm{C}$, write $0 \times \mathrm{BB}$, write $0 \times 27$

Repeated start, write 0xB7

Read LSB \&MSB

Convert temperature (T) to ${ }^{\circ} \mathrm{C}$

Compute averaged $\mathrm{T}\left({ }^{\circ} \mathrm{C}\right)$

Write temperature to UART

Status LED =True

Delay 500ms

End IF

Status LED =False

End Main Loop

In this algorithm, the temperature value is measured 3-times to make a simple moving average filtering, and the filtered value $T\left({ }^{\circ} C\right)=\left(T_{1}+T_{2}+T_{3}\right) / 3$ is sent to a smart phone or tablet.

\subsection{Android Based Monitoring Application}

An Android-based monitoring application has been developed to monitor the temperature data in the system and to control the measurement devices. The main objective of the application is to monitor the temperature values measured in real-time by the measuring device and to warn the user when a temperature above the determined threshold value is detected. Furthermore, the application has functionalities such as measurement device selection, threshold temperature value configuration and temperature calibration. Data communication between the application and the measurement device is provided via Bluetooth, which is a communication protocol that stands out with its low power consumption and widespread use. The flowchart of the monitoring application is outlined in Figure 4. 


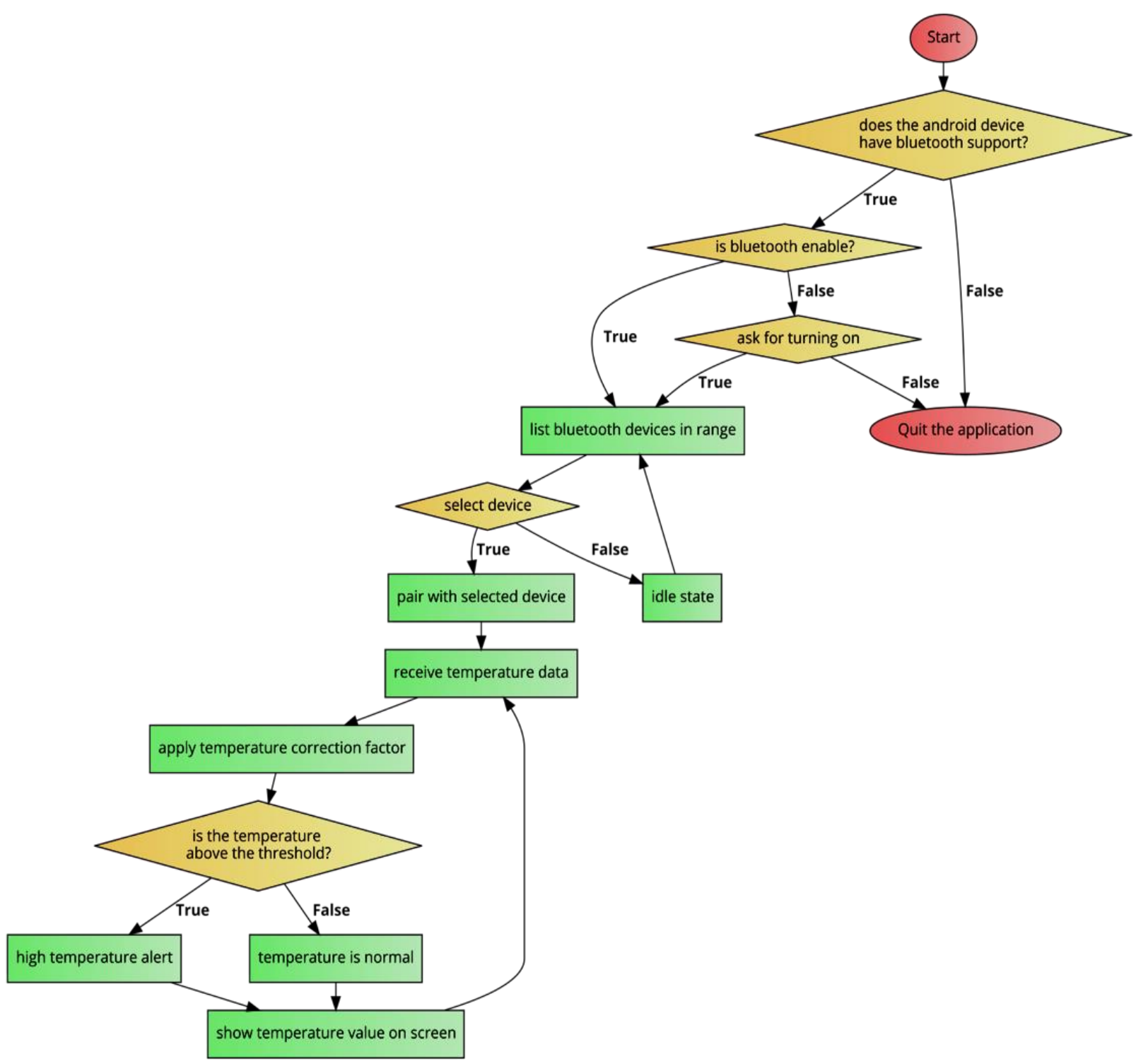

Figure 4. The flowchart for Android-based monitoring application

When the application is opened, it first scans the Bluetooth devices in range and presents the list of available devices to the user for selection. After the device selection, a virtual serial port interface is established over Bluetooth to be used for data transfer. With the start command sent from the application, the measuring device starts to send the measured temperature data in the specified period. In the temperature data, the threshold evaluation process is started after the temperature correction determined in accordance with the region where the temperature is measured. Temperature correction value can be modified with the "Calibration" option in the application settings. If the obtained temperature value is above the determined maximum value, the high-temperature phrase appears on the application screen and the user is warned audibly. Otherwise, only the temperature value on the application screen is updated. Using the application settings menu, the threshold value for the maximum temperature can be modified, and the warning function can be activated for cases where the threshold value is exceeded. As long as the application is open, the temperature evaluation process continues cyclically. 


\section{Results and Discussion}

First, we have designed an electronic circuit to measure and transmit temperature value. The PIC12LF1822 microcontroller, HC-05 Bluetooth v2, and MLX 90615 IR non-contact temperature sensor-based circuit has been integrated, and LDR triggering is adopted to its algorithm. After the wrist is closed to the device, contactless measurement starts, and the temperature value is sent to an Android device. The schematic of the circuit is shown in Figure 5 .

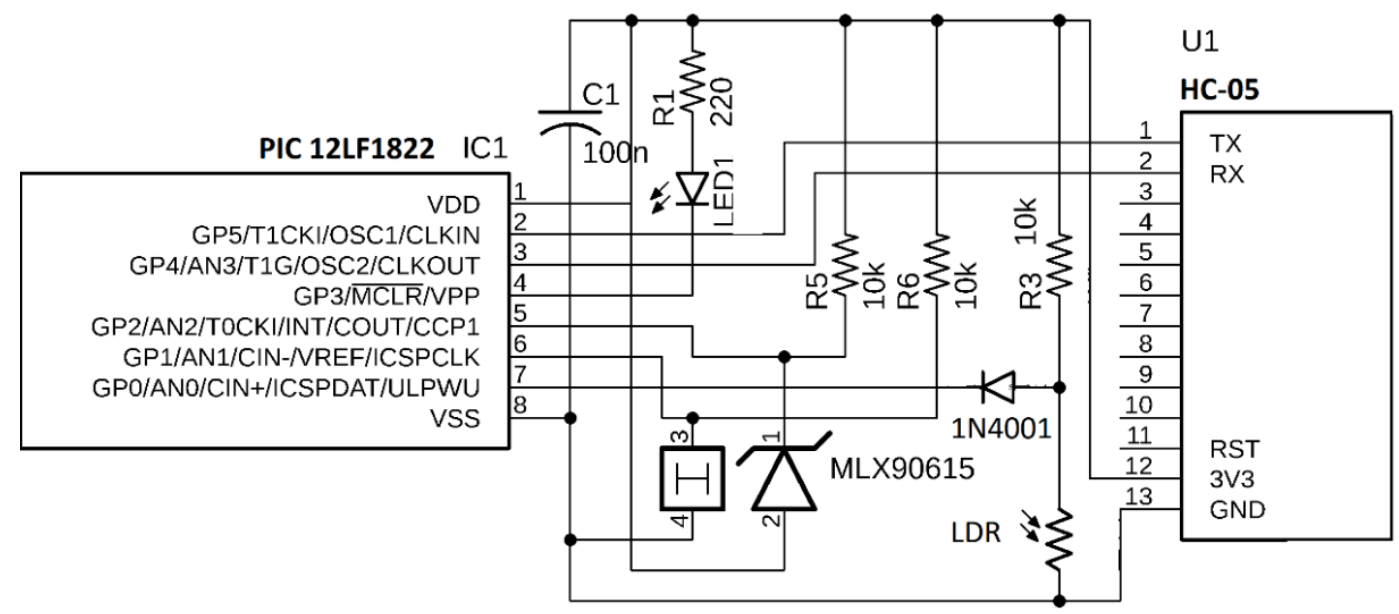

Figure 5. The schematic view of the microcontroller-based circuit.

C language-based approximately $2 \mathrm{kB}$ code is developed for this device. SMbus (I2C) and UART protocols, and the conversions are embedded. For user-friendly usage of the device, the 3-D model of the chassis is designed. This model and the position of the temperature measurement from the wrist are shown in Figure 6.

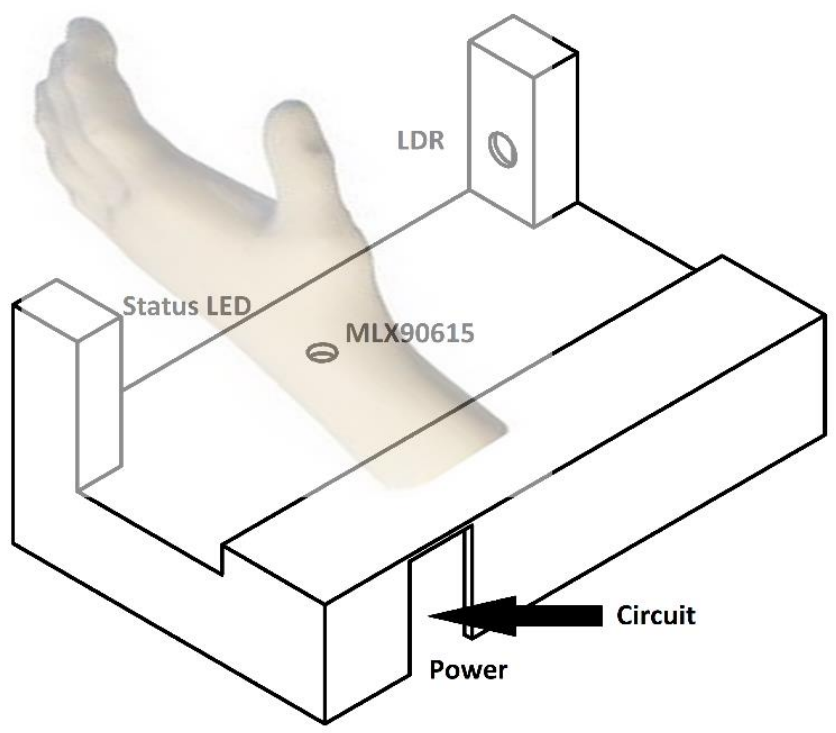

Figure 6. 3-D chassis model and the scheme of the measurement from the wrist. 
3D model is then constructed using a 3D printer, and the circuit is mounted in it. After the user puts the wrist to closer to LDR, at the same time, closer to MLX90615 sensor, it is measured and sent to the application in on a smartphone from long distance. The Android application can be seen in Figure 7.

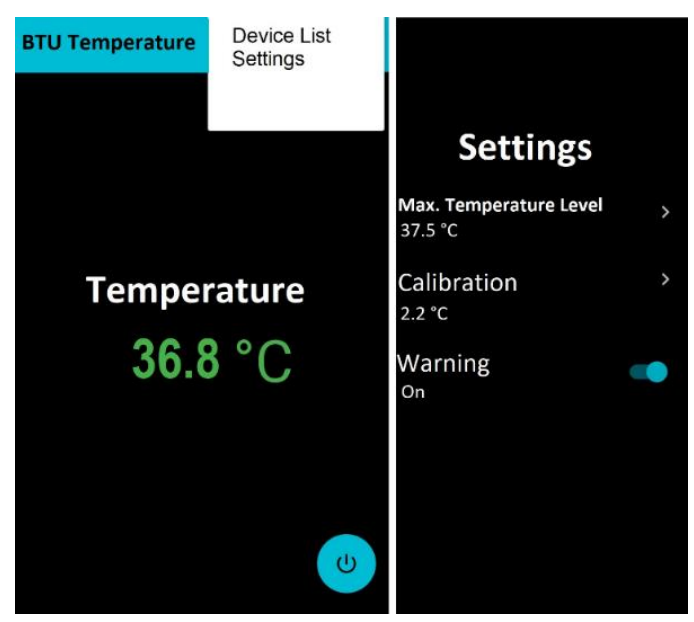

Figure 7. The view of the Android application.

The transferred measurement data to the application is in object mode temperature. In other words, it should be arranged to body temperature considering measurement from the wrist. For this reason, we measured the temperature of the wrist at object mode, and then body mode at least 10 times using a calibrated device. The mean difference between object and body mode $\left(2.2^{\circ} \mathrm{C}\right)$ is added to the calibration panel on the application. This can be also re-adjusted if any deviation exists.

With the help of the designed device and application, body temperature in the entrance of the university or mall can be detected from long distance in accordance with isolation in a pandemic situation. In case of fever, audible and visual alarms can be set.

This is a low-cost design for checking body temperature without getting closer, however, it has limitations of the range and voice. To compare the study to previous designs, their technologies are summarized in Table 1.

Table 1: The Comparison of Body Temperature Measurement Devices in the Literature.

\begin{tabular}{ll}
\hline Study & Technology \\
\hline Kuzu and Tulum [4] & Atmega, SpO2, blood pressure, temperature (only measurement) \\
Şakar and Öniz [5] & PIC18F4620, DS18B20, MLX90614 (Contact/non-contact sensor) \\
Gülenç \& Kartal [8] & Atmega328P, Max30100, MLX90614, MPU6050, ESP8266, WEB \\
Korkmaz et al. [10] & PIC16F877A, LabVIEW, Nonin 800A, DS18B20, RF UFM-M11 \\
Wijaya et al [12] & Atmega16, LM35, Heart rate (only measurement) \\
Gupta et al. [13] & Arduino, Heart Rate, NTC, LCD \\
Mert et al. [14] & PIC18F4550, LCD, MLX90615, HIH6006, DBM01, Wi-Fi. \\
Hasan [16] & MAX30205, ESP8266 MCU, SR-04, RFID (RC522), ThingSpeak, IoT \\
Karthi \& Jayakumar [18] & NodeMCU ESP8266, AWS IoT,RFID, DT11, GPS \\
This study & MLX90615, PIC12LF1822, LDR, HC-05, Android application. \\
\end{tabular}


This design in this paper is an effective and low-cost solution considering the pandemic issues. HC-05 Bluetooth v2.0 has a wider range (up to $10 \mathrm{~m}$ ) when compared to Bluetooth 4.0 low energy modules, but it might be insufficient for some places. Moreover, PIC12LF1822 is suitable to re-design it using battery due to low power consumption, but it has $2 \mathrm{kB}$ flash program memory, which disables us to add voice command option and speaker. For further designs, these considerations can be re-designed.

\section{Conclusion}

In this study, we have designed a low-cost microcontroller-based body temperature measurement circuit with an Android application. Infrared non-contact temperature sensor is connected to microcontroller using I2C protocol, and Bluetooth module at the UART pins is used to transfer body temperature. With the help of developed Android application, the received data from a smart telephone or tablet can be shown, checked, and audible or visual alarms can be set in case of fever. Thus, long-distance measurement circuit with its microcontroller and application codes are developed considering the COVID-19 pandemic conditions. At the entrance of a building such as a university or a mall, the fever of the visitors can be detected safely, without contacting physically.

\section{Acknowledgement}

This work is supported by the Research Fund of Bursa Technical University, Project Number: 200COVID05.

\section{References}

[1] Hanilçi, A., \& Gürkan, H. (2019). ECG biometric identification method based on parallel 2-D convolutional neural networks. Journal of Innovative Science and Engineering (JISE), 3(1), 11-22.

[2] Verma, S., \& Gupta, N. (2012). Microcontroller-based Wireless Heart Rate Telemonitor for Home Care. IOSR J Eng (IOSRJEN), 2(7), 25-31.

[3] Castaneda, D., Esparza, A., Ghamari, M., Soltanpur, C., \& Nazeran, H. (2018). A review on wearable photoplethysmography sensors and their potential future applications in health care. International journal of biosensors \& bioelectronics, 4(4), 195.

[4] Kuzu, M., Taş, O., \& Tulum, G. (2017). Uzaktan İzlenebilir Hasta Parametreleri Sistemi. 2. Ulusal Biyomedikal Cihaz Tasarımı ve Üretimi Sempozyumu, 16 Mayıs 2017.

[5] Özgören, M., Şakar, M., \& Öniz, A. (2010,). Contact/non-contact sensor mesh for body temperature monitoring. In 2010 15th National Biomedical Engineering Meeting (pp. 1-4). IEEE.

[6] Chung, Y. T., Yeh, C. Y., Shu, Y. C., Chuang, K. T., Chen, C. C., Kao, H. Y., Ko, W. C., Chen, P. L., \& Ko, N. Y. (2020). Continuous temperature monitoring by a wearable device for early detection of febrile events in the SARS-CoV-2 outbreak in Taiwan, 2020. Journal of microbiology, immunology, and infection 53(3), 503504. https://doi.org/10.1016/j.jmii.2020.04.005 
[7] Holt, S. G., Yo, J. H., Karschimkus, C., Volpato, F., Christov, S., Smith, E. R., ... \& Champion De Crespigny, P. (2020). Monitoring skin temperature at the wrist in hospitalised patients may assist in the detection of infection. Internal Medicine Journal.

[8] Gülenç, N. G., \& Kartal, M. (2020, November). Noninvasive Measurement of Baby's Vital Data and Mobile Monitoring-Analysis System Design. In 2020 Medical Technologies Congress (TIPTEKNO) (pp. 1-4). IEEE.

[9] Duman, Ü., \& Aydin, E. (2020, September). IOT Based Baby Cradle System with Real Time Data Tracking. In 2020 5th International Conference on Computer Science and Engineering (UBMK) (pp. 274-279). IEEE.

[10] Fidan, U., \& Güler, N. F. (2007). 4 Kanallı Biyotelemetri Cihazı Tasarımı. Journal of the Faculty of Engineering \& Architecture of Gazi University, 22(1).

[11] Eriş, Ö., Korkmaz, H., Toker, K., \& Buldu, A. (2010). İnternet Üzerinden Hasta Takibi Amaçlı PIC Mikrodenetleyici Tabanlı Kablosuz Pals-Oksimetre Ölçme Sistemi Tasarımı ve LabVIEW Uygulaması. TURKMIA, 10, 16-25.

[12] Wijaya, N. H., Fauzi, F. A., Helmy, E. T., Nguyen, P. T., \& Atmoko, R. A. (2020). The Design of Heart Rate Detector and Body Temperature Measurement Device Using ATMega16. Journal of Robotics and Control (JRC), 1(2), 40-43.

[13] Gupta, S., Talwariya, A., \& Singh, P. (2020). Development of Arduino-Based Compact Heart Pulse and Body Temperature Monitoring Embedded System for Better Performance. In Performance Management of Integrated Systems and its Applications in Software Engineering (pp. 189-197). Springer, Singapore.

[14] Mert, A., Seçgin, Ö., \& Akan, A. Sürekli Vucut Sıcaklıgı Ölçümü Için Biyotelemetri Cihaz Tasarımı Tıp Teknolojileri Ulusal Kongresi, TIPTEKNO’14 (pp.312-315). Nevşehir, Turkey

[15] Kim, Y., Lee, S., \& Lee, S. (2015). Coexistence of ZigBee-based WBAN and WiFi for health telemonitoring systems. IEEE journal of biomedical and health informatics, 20(1), 222-230.

[16] Hasan, M. W. (2021). Covid-19 fever symptom detection based on IoT cloud. International Journal of Electrical \& Computer Engineering, 11(2), 1823-1829.

[17] Akleylek, S, Kılıç, E, Söylemez, B, Aruk, E, Aksaç, C. (2020). Nesnelerin İnterneti Tabanlı Sağlık İzleme Sistemleri Üzerine Bir Çalışma. Mühendislik Bilimleri ve Tasarım Dergisi, Special Issue: International Conference on Artificial Intelligence and Applied Mathematics in Engineering (ICAIAME 2020), 80-89.

[18] Karthi, P., Jayakumar, M. (2020). Smart Integrating Digital Contact Tracing with IoMT for COVID-19 using RFID and GPS. Journal of Xi' an Shiyou University, Natural Science Edition, 16(12), 38-43.

[19] Costin, H., \& Rotariu, C. (2018). Vital Signs Telemonitoring by Using Smart Body Area Networks, Mobile Devices and Advanced Signal Processing. In Advances in Biomedical Informatics (pp. 219-246). Springer, Cham.

[20] Melexis MLX 90615 temperature sensor datasheet, https://www.melexis.com//media/files/documents/datasheets/mlx90615-datasheet-melexis.pdf, 30.11.2020.

[21] PIC12LF1822 microcontroller datasheet, http://ww1.microchip.com/downloads/en/devicedoc/40001413e.pdf, 30.11. 2020. 